\title{
Optical and microwave control of germanium-vacancy center spins in diamond
}

\author{
Petr Siyushev, ${ }^{1, *}$ Mathias H. Metsch, ${ }^{1}$ Aroosa Ijaz, ${ }^{1}$ Jan M. Binder, ${ }^{1}$ Mihir K. Bhaskar, ${ }^{2}$ Denis D. Sukachev, ${ }^{2,3}$ Alp Sipahigil, ${ }^{2}$ \\ Ruffin E. Evans, ${ }^{2}$ Christian T. Nguyen, ${ }^{2}$ Mikhail D. Lukin, ${ }^{2}$ Philip R. Hemmer, ${ }^{4}$ Yuri N. Palyanov, ${ }^{5,6}$ Igor N. Kupriyanov, ${ }^{5,6}$ \\ Yuri M. Borzdov, ${ }^{5,6}$ Lachlan J. Rogers, ${ }^{1, \dagger}$ and Fedor Jelezko ${ }^{1,7}$ \\ ${ }^{1}$ Institute for Quantum Optics, Ulm University, D-89081 Ulm, Germany \\ ${ }^{2}$ Department of Physics, Harvard University, 17 Oxford Street, Cambridge, Massachusetts 02138, USA \\ ${ }^{3}$ P. N. Lebedev Physical Institute of the RAS, Moscow 119991, Russia \\ ${ }^{4}$ Electrical \& Computer Engineering Department, Texas A\&M University, College Station, Texas 77843, USA \\ ${ }^{5}$ Sobolev Institute of Geology and Mineralogy, Siberian Branch of Russian Academy of Sciences, \\ Koptyug Avenue 3, Novosibirsk 630090, Russia \\ ${ }^{6}$ Novosibirsk State University, Novosibirsk 630090, Russia \\ ${ }^{7}$ Center for Integrated Quantum Science and Technology (IQ $\left.{ }^{\text {st }}\right)$, Ulm University, D-89081 Ulm, Germany
}

(Received 12 December 2016; published 18 August 2017)

\begin{abstract}
A solid-state system combining a stable spin degree of freedom with an efficient optical interface is highly desirable as an element for integrated quantum-optical and quantum-information systems. We demonstrate a bright color center in diamond with excellent optical properties and controllable electronic spin states. Specifically, we carry out detailed optical spectroscopy of a germanium-vacancy $(\mathrm{GeV})$ color center demonstrating optical spectral stability. Using an external magnetic field to lift the electronic spin degeneracy, we explore the spin degree of freedom as a controllable qubit. Spin polarization is achieved using optical pumping, and a spin relaxation time in excess of $20 \mu \mathrm{s}$ is demonstrated. We report resonant microwave control of spin transitions, and use this as a probe to measure the Autler-Townes effect in a microwave-optical double-resonance experiment. Superposition spin states were prepared using coherent population trapping, and a pure dephasing time of about 19 ns was observed at a temperature of $2.0 \mathrm{~K}$.
\end{abstract}

DOI: 10.1103/PhysRevB.96.081201

Over the last few decades significant effort has been directed towards the exploration of solid-state atomlike systems such as quantum dots or color centers in diamond owing to their potential application in quantum-information processing [1-4]. The nitrogen-vacancy (NV) center in diamond has become prominent due to its optical spin initialization and readout [5], and the ease of spin control by microwave fields [1]. However, the small Debye-Waller factor of this defect [6] and its spectral instability [7] hinder the realization of an efficient quantum-optical interface [8], motivating an ongoing search for new candidates. Here, we investigate the recently discovered germanium-vacancy $(\mathrm{GeV})$ center in diamond [9-11], demonstrating its outstanding spectral properties devoid of measurable spectral diffusion. We show spin- $\frac{1}{2}$ Zeeman splitting which confirms this is the negative charge state of this defect. We use two-photon resonance to optically prepare coherent dark spin superposition states, and show direct spin manipulation via resonant microwave fields. The spin coherence time is found to be $T_{2}^{\star}=19 \pm 1 \mathrm{~ns}$, which is concluded to be limited by phonon-mediated orbital relaxation as in the closely related silicon-vacancy (SiV) center [12-14]. Optical and microwave control of GeV spin, combined with the possibility of $\mathrm{GeV}$ centers in nanophotonic

\footnotetext{
*petr.siyushev@uni-ulm.de

†lachlan.j.rogers@quantum.diamonds
}

Published by the American Physical Society under the terms of the Creative Commons Attribution 4.0 International license. Further distribution of this work must maintain attribution to the author(s) and the published article's title, journal citation, and DOI. devices [15], make it a promising platform for quantum optics and quantum-information science applications.

The GeV center can be produced in diamond during crystal growth and by ion implantation, and it fluoresces strongly with a zero-phonon line at $602 \mathrm{~nm}$ accompanied by a weak phonon sideband (PSB) containing about $40 \%$ of the fluorescence $[9,10]$. Isotopic shifts of the fluorescence spectrum established that the defect contains a single germanium atom $[11,16]$, and $a b$ initio calculations suggest that it is formed by the Ge atom taking the place of two carbon atoms and relaxing its position to split the adjacent vacancy $[9,17]$. The resulting geometry is aligned along a $\langle 111\rangle$ axis and has inversion-symmetric $\mathrm{D}_{3 \mathrm{~d}}$ symmetry, as illustrated in Fig. 1(a). This structure is identical to the SiV center in diamond $[18,19]$ and leads to ${ }^{2} E_{g}$ and ${ }^{2} E_{u}$ ground and excited states, respectively. These have twofold degeneracy in both spin and orbit, which is partially lifted by spin-orbit interactions to produce levels labeled 1 , 2 (ground) and 3, 4 (excited) in order of increasing energy. Transitions between these lead to a four-line fine structure of the zero-phonon line (ZPL), as shown in Fig. 1(a) [10].

The experiments were performed on two identical $2 \times 2 \times 1$ $\mathrm{mm}$ samples polished in the $\{100\}$ and $\{111\}$ planes. These diamonds were grown by high-pressure high-temperature synthesis in a Mg-Ge-C system, and the $\mathrm{GeV}$ centers were incorporated during this process $[10,16]$. Solid immersion lenses (SILs) were fabricated on the diamonds by focused ion beam milling [Fig. 1(b)] to increase fluorescence collection efficiency [20-22]. Individual $\mathrm{GeV}$ defects were excited resonantly by a tunable dye laser and addressed in a confocal microscope at a cryogenic temperature $(T \approx 2.0 \mathrm{~K})$, as shown in Fig. 1(c). An optical long-pass filter with a cut-on edge at $610 \mathrm{~nm}$ was placed in the detection channel to reject scattered 


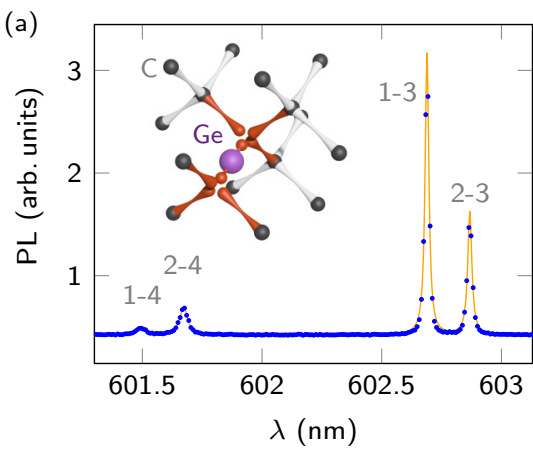

(b)

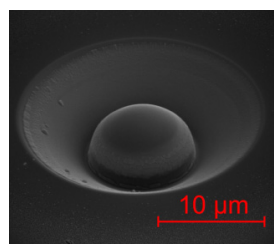

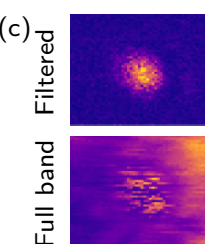

जn $(d)$

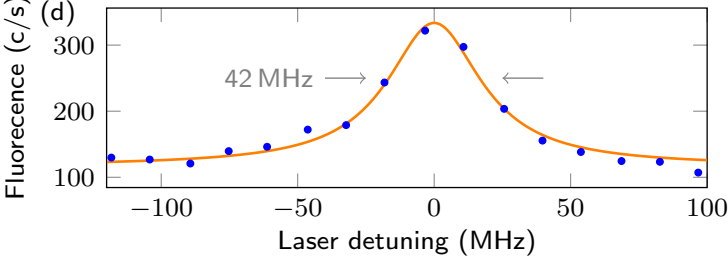

(f)

\section{$\frac{n}{n}$}

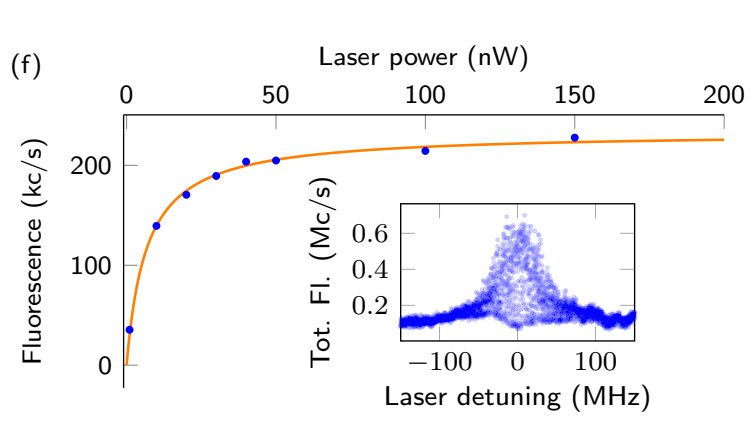

(e)

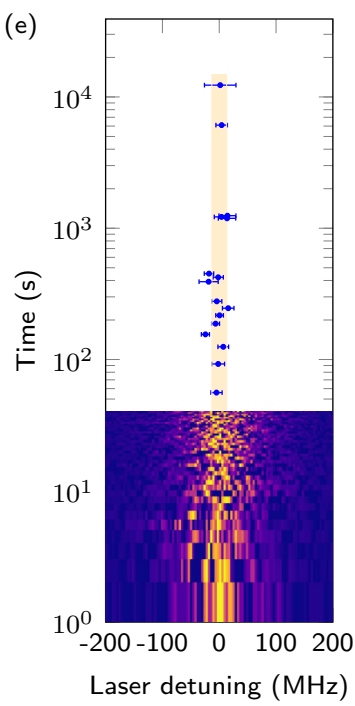

FIG. 1. Stable narrow optical transitions. (a) PL spectrum of GeV at $35 \mathrm{~K}$, demonstrating the four-line ZPL structure. Inset: Molecular structure of the $\mathrm{GeV}$ center in diamond showing a Ge atom taking the place of two adjacent carbon atoms. (b) Scanning electron microscopy (SEM) image of a solid immersion lens fabricated on the sample surface. (c) Fluorescence image of a single GeV center located off center under the SIL, recorded with a long-pass filter (sideband only) and without any filter on the detector. (d) In the low-intensity limit, linewidths as narrow as $42 \mathrm{MHz}$ were recorded. (e) The GeV transitions were stable in frequency for measurements of several hours. (f) Saturation curve for a single $\mathrm{GeV}$ center recorded under resonant excitation (only photons from the phonon sideband are recorded). The inset shows a single-sweep PLE spectrum recorded without a long-pass filter (entire emission band is recorded), yielding up to $0.6 \mathrm{Mc} / \mathrm{s}$ at saturation intensity.

laser light, meaning that only photons associated with the phonon sideband were detected.

A resonant laser was scanned across the transition between the lower branches of the ground and excited state (transition 1-3) and a linewidth of $42 \mathrm{MHz}$ was measured, as shown in Fig. 1(d). This is less than double the $26 \mathrm{MHz}$ transform limit imposed by the excited state lifetime of 6 ns [15], indicating the stability of the transition over the measurement duration of $40 \mathrm{~s}$. Subsequent measurements at intervals over $4 \mathrm{~h}$ demonstrated the line to be stable at longer time scales [Fig. 1(e)].

The linewidth and stability were probed at lower laser excitation intensities to avoid power broadening. Figure 1(f) presents a saturation measurement on the optical transition, yielding more than $200 \mathrm{kc} / \mathrm{s}$ detected in the sideband. The off-center position of this $\mathrm{GeV}$ center in the SIL meant that laser scatter was collected only weakly, allowing a signal-to-noise ratio of 5:1 even after removing the filter from the detection path [Fig. 1(c)]. Photoluminescence excitation (PLE) spectra are typically measured on a carefully filtered sideband to eliminate the dominant laser scatter. The high contrast observed here without any filter is evidence of a strong optical transition. Collecting the whole fluorescence band in this manner yielded up to $0.6 \mathrm{Mc} / \mathrm{s}$, as shown inset to Fig. 1(f). These data exhibit a blinking phenomenon that was observed to intensify for higher excitation intensities. Blinking has also been observed for $\mathrm{SiV}$ centers in certain diamond samples $[23,24]$ but not others [19]. It is therefore anticipated that blinking is not intrinsic to the $\mathrm{GeV}$ center itself and can be controlled using superior sample preparation techniques.

The $\{100\}$ sample was mounted over neodymium magnets producing a field of about $0.3 \mathrm{~T}$ aligned roughly in the plane of the diamond surface, as illustrated in Fig. 2(a). Figures 2(b) and 2(c) show the Zeeman-split PLE spectra for transitions 1-3 and 2-3 of an individual $\mathrm{GeV}$ center. Various observations presented below indicate that this $\mathrm{GeV}$ center was aligned almost perpendicular to the field. For this center the ground state splitting between levels 1 and 2 was found to be about $170 \mathrm{GHz}$, which is about $20 \mathrm{GHz}$ larger than the minimum (intrinsic) ground state splitting [15]. This additional lifting of the orbital degeneracy is attributed to the presence of transverse strain. The measurement temperature of $2.0 \mathrm{~K}$ was cold enough to reduce the thermal population in level 2 compared to level 1 , and so the absolute intensity is less for transition 2-3 than for 1-3. Transitions to state 4 are of less interest because of rapid thermalization into state 3 [14], and were not measured here in PLE. The fourfold Zeeman splitting patterns suggest electronic spin- $\frac{1}{2}$ splitting of each of the states, as illustrated in Fig. 2(d). This is consistent with the $602 \mathrm{~nm}$ band arising from the negative charge state of $\mathrm{GeV}$.

This Zeeman-split situation can be modeled using the Hamiltonian developed for the similarly structured $\mathrm{SiV}$ center [18]. Quantitative analysis is not possible since our experiment design prevents an accurate understanding of the field magnitude and orientation at the $\mathrm{GeV}$ center. However, the spectra in Fig. 2(a) indicate that levels 1 and 2 have almost identical Zeeman splitting (here $1530 \mathrm{MHz}$ ). Modeling suggests that the magnetic field is highly misaligned, in which case the splitting is dictated not by the Zeeman effect but by transverse strain (in the absence of strain the transverse magnetic field cannot lift the spin degeneracy).

Since optical transitions are spin conserving, the stronger (weaker) peaks in Figs. 2(b) and 2(c) correspond to transitions between states with more (less) similar spin projection. For convenience we describe the two kinds of transitions as "spin conserving" and "spin flipping", respectively. The applied 
(a)

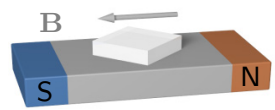

(b)

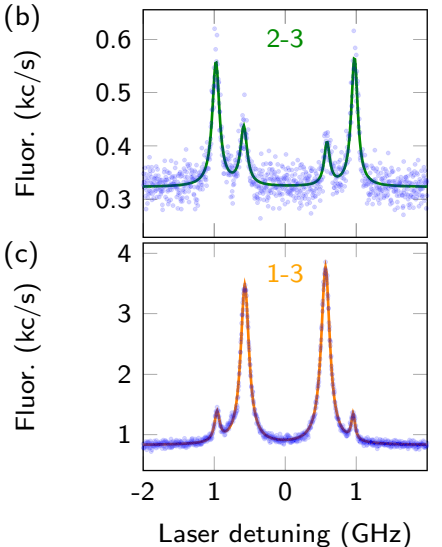

(d)

$$
E_{\mathrm{u}} \underset{i}{\mathbb{S}^{\prime}}
$$

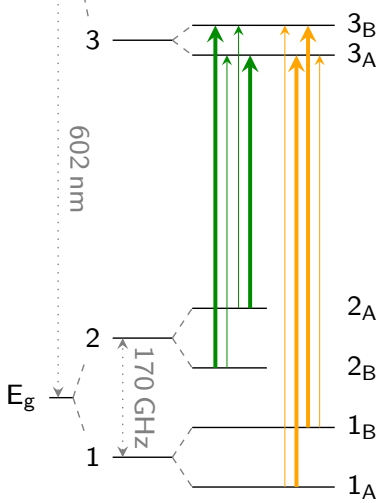

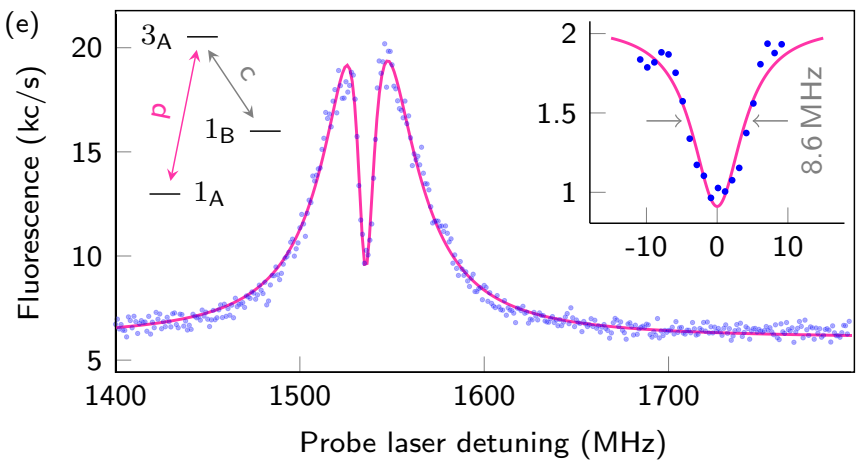

FIG. 2. Optical lambda schemes. (a) The diamond sample was mounted over neodymium magnets, giving a field in the plane of the $\{100\}$ surface. (b) PLE spectrum shows transition 2-3 split into four peaks, with the outer two being strongest. (c) PLE spectrum shows transition 1-3 split into four peaks, with the inner two being strongest. (d) The magnetic field lifts the spin degeneracy and produces doublets from each of the ground and excited state branches ( $\mathrm{A}$ and $\mathrm{B}$ subscripts describe two spin states). (e) $\mathrm{CPT}$ was performed on the $1_{\mathrm{A}}-3_{\mathrm{A}}-1_{\mathrm{B}} \Lambda$ scheme, demonstrating coherent optical spin manipulation. The inset depicts the narrowest dip, which was found to be $8.6 \pm 0.5 \mathrm{MHz}$ wide corresponding to a coherence lifetime of $19 \pm 1 \mathrm{~ns}$

magnetic field component transverse to the $\langle 111\rangle$ symmetry axis leads to different spin quantization axes in the ground and excited states [4], resulting in the spin-flipping transitions being visible in the PLE spectra. Interestingly, the conserving and flipping transitions have inverted order for transition 2-3 compared to $1-3$. This detail is in contrast to the observations for the $\mathrm{SiV}$ center [12], and does not arise from the model Hamiltonian under strain or magnetic field misalignment. It warrants future investigation, although it does not influence the coherent spin manipulations reported here (which utilize the $1_{\mathrm{A}}$ and $1_{\mathrm{B}}$ levels).

The presence of all four lines in the Zeeman-split spectrum of transition 1-3 indicates that lambda schemes are accessible optically. These provide an opportunity to investigate coherence properties through optically prepared superpositions of the ground states [25]. We make use of coherent population trapping $(\mathrm{CPT})$, in which a dark superposition state is produced

when driving optical fields are resonant to both transitions of the $\Lambda$ scheme. The state is dark due to quantum interference, resulting in a dip in the excitation spectrum with a width limited by the lifetime of the superposition. Both excitation frequencies for CPT were generated from a single laser using a high-bandwidth electro-optic amplitude modulator with the carrier on transition $1_{\mathrm{B}}-3_{\mathrm{A}}$ as the pump, and a sideband tuned across transition $1_{\mathrm{A}}-3_{\mathrm{A}}$ as the probe. The CPT linewidths in Fig. 3(b) are therefore insensitive to laser frequency noise, and the narrowest dip width observed was $8.6 \pm 0.5 \mathrm{MHz}$, corresponding to a coherence time of $19 \pm 1 \mathrm{~ns}$.

The optical $\Lambda$ schemes identified in the $\mathrm{GeV}$ electronic structure also provide a mechanism for polarizing the spin. Resonantly exciting the spin-conserving transition $1_{\mathrm{A}}-3_{\mathrm{A}}$ led to optical pumping into the spin-B levels of the ground state, as illustrated in Fig. 3(a). Time-resolved fluorescence measurements indicate an optical pumping contrast of 59\%, which is the spin polarization [normalized population difference $\left.\left(P_{\mathrm{B}}-P_{\mathrm{A}}\right) / P_{\text {total }}\right]$ [26]. This spin polarization is a function of the optical pumping rate compared to the spin relaxation rate, and can be increased with higher laser powers. Since it is necessary to spectrally resolve the Zeeman-split transitions, excitation intensity (and hence spin polarization) is limited by the need to avoid power broadening beyond the Zeeman splitting.

A $25 \mu \mathrm{m}$ wire placed across the sample surface was used to apply microwaves at a frequency of $1530 \mathrm{MHz}$ corresponding to the $1_{\mathrm{A}}-1_{\mathrm{B}}$ transition, and a sharp increase in the steady-state
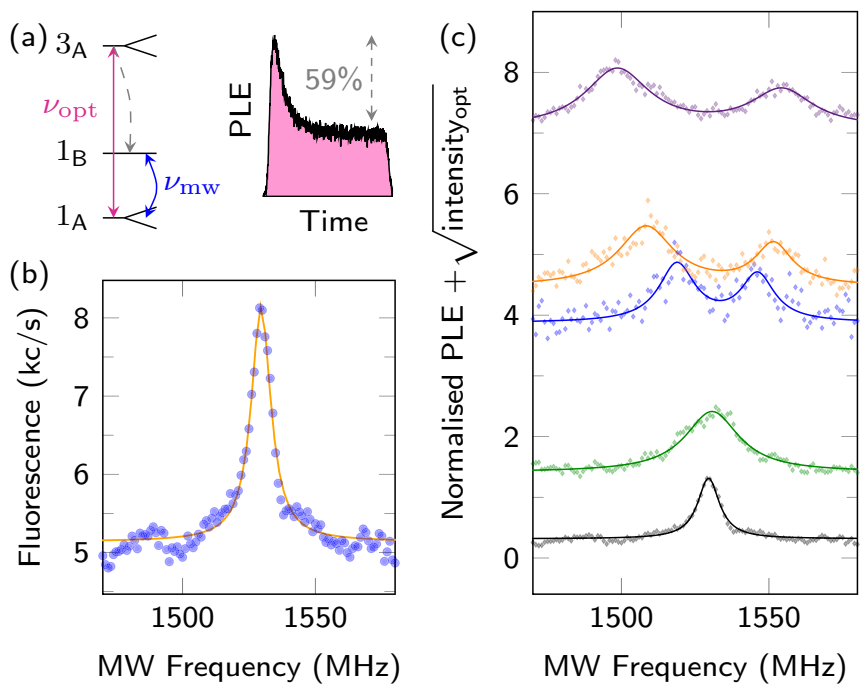

FIG. 3. Microwave manipulation of GeV spin. (a) Excitation of the spin-conserving transition $1_{\mathrm{A}}-3_{\mathrm{A}}$ polarizes the spin due to optical pumping into $1_{\mathrm{B}}$. The time-resolved fluorescence for an excitation pulse shows spin polarization of 59\% (limited by laser intensity). High optical Rabi frequencies lead to Autler-Townes splitting of levels $1_{\mathrm{A}}$ and $3_{\mathrm{A}}$. (b) Microwaves resonant to $1_{\mathrm{A}}-1_{\mathrm{B}}$ return the population to $1_{\mathrm{A}}$ and restore the higher fluorescence. The width of the microwave transition is $9 \mathrm{MHz}$ corresponding to a dephasing time of about $19 \mathrm{~ns}$. The microwave-transition contrast of about $35 \%$ is limited by the $59 \%$ contrast possible from spin polarization. (c) The microwave transition splits at increased laser intensity. The splitting scales linearly with the square root of laser intensity, as expected for the Autler-Townes effect. 


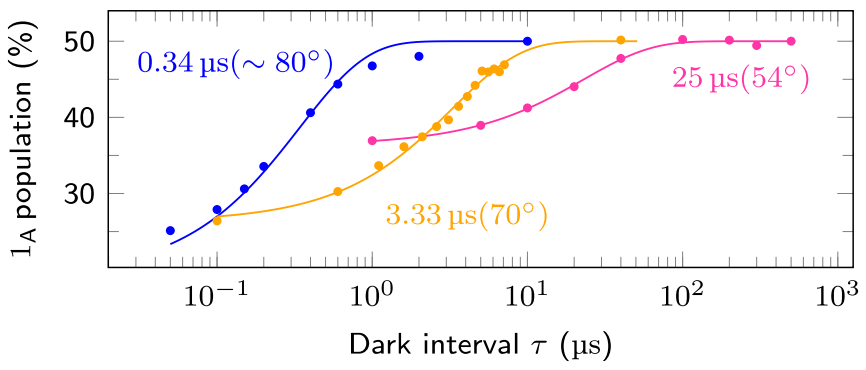

FIG. 4. Spin relaxation. Pulsed excitation was used to polarize the spin into $1_{\mathrm{B}}$ via optical pumping as in Fig. 3(a). The dark interval $\tau$ between laser pulses was varied to obtain the spin relaxation time (lines are exponential fits). For the $\sim 80^{\circ}$ field misalignment in the optically detected magnetic resonance (ODMR) and CPT measurements, $T_{1}=0.34 \mu \mathrm{s}$ was measured. Improving the field alignment produced longer spin relaxation times $\left(3.33 \mu \mathrm{s}\right.$ at $70^{\circ}$ and $25 \mu \mathrm{s}$ at $54^{\circ}$ ) but lower spin polarization.

fluorescence was observed [Fig. 3(b)]. This occurs due to the microwaves driving a spin transition within the ground state that returns the population to the $1_{\mathrm{A}}$ level. Such microwave access to this spin transition in the $\mathrm{GeV}$ and $\mathrm{SiV}$ systems provides a simple and direct way to manipulate the spin state. Avoiding power broadening on the microwave and optical transitions produced a microwave-spectrum linewidth of $9.0 \pm 0.5 \mathrm{MHz}$ [Fig. 3(b)], corresponding to a dephasing time of $19 \pm 1 \mathrm{~ns}$. The microwave-driven contrast of about $35 \%$ is limited by the spin polarization.

The microwave resonance occurred at a frequency exactly matching the two-photon detuning for laser fields producing CPT [Fig. 2(e)], proving that the fluorescence response is due to microwave manipulation of the ground state levels $1_{\mathrm{A}}$ and $1_{\mathrm{B}}$. The microwave-transition linewidth and CPT dip width were also identical within the accuracy of the measurement, presenting a consistent picture regarding the coherence time of the Zeeman-split levels. Both of these quantities are fundamentally limited by the phase lifetime of the $1_{\mathrm{A}}$ and $1_{\mathrm{B}}$ ground states.

Increasing the resonant excitation intensity on the optical transition $1_{\mathrm{A}}-3_{\mathrm{A}}$ led to a broadening and then splitting of the microwave-transition peak, as shown in Fig. 3(c). The splitting increased proportionally to the square root of laser intensity, confirming that it arises due to the optical Rabi frequency. For optical Rabi oscillations faster than the excited state lifetime, dressed states are resolvable, and the weak microwave field acts as a probe to measure this splitting. This observation of the Autler-Townes effect indicates that coherent Rabi oscillations are achieved on the optical transition for excitation intensities above saturation. It provides further evidence of the stable narrow optical transition for $\mathrm{GeV}$.

Spin relaxation was measured by varying the dark intervals between the $1_{\mathrm{A}}-3_{\mathrm{A}}$ excitation pulses of Fig. 3(a), yielding $T_{1}=0.3 \mu \mathrm{s}$, as shown in Fig. 4(a). Other field alignments were achieved by placing the sample flat on a pole of a disk magnet, producing a field normal to the sample surface. For the $\{111\}$ sample a number of $\mathrm{GeV}$ centers were measured to have $T_{1}=3.3 \pm 0.3 \mu \mathrm{s}$. The $\{100\}$ sample gives a $\mathrm{GeV}$ misalignment of only $54^{\circ}$, and the spin relaxation time was measured to be $T_{1}=25 \pm 5 \mu \mathrm{s}$. These results are shown in Fig. 4. For SiV centers it was observed that spin $T_{1}$ extends for better aligned fields [12], and the same phenomenon is exhibited here. It is concluded that the $\mathrm{GeV}$ centers measured in the $\{111\}$ sample had a misalignment of $70^{\circ}$, and that the arbitrary field used for the double-resonance measurements was misaligned by more than $80^{\circ}$. Improved field alignment increases the spin relaxation time, but it also means the optical transition selection rules become more exclusive to the spinconserving transitions. It was therefore easier to achieve spin polarization for a misaligned field, as shown by the equilibrium $1_{\mathrm{A}}$ population $(\tau \rightarrow 0$ ) in Fig. 4 , and this effect prevented a measurement of $T_{1}$ for the aligned $\mathrm{GeV}$ centers in the $\{111\}$ sample.

The double-resonance measurements indicate that levels $1_{\mathrm{A}}$ and $1_{\mathrm{B}}$ have a coherence time of about $19 \mathrm{~ns}$, which is considerably shorter than the spin $T_{1}$. The situation is most readily interpreted by analogy with the closely related negatively charged $\mathrm{SiV}$ center. For $\mathrm{SiV}$ it was found that resonant phonons mediated orbital relaxation on a fast time scale of about $40 \mathrm{~ns}$ at $5 \mathrm{~K}$, and this limited the spin coherence time [12-14]. This occurs because a different spin detuning for each orbital eigenstate means that coherent spin states rapidly acquire a large unknown phase after an orbital flip. It seems that the same process occurs in the GeV center, however, for similar phonon coupling parameters, the orbital relaxation rate will be even faster as a result of the increased spin-orbit splitting in the ground state. These energies are within the Debye approximation regime for diamond, and so the phonon density of states increases with energy. This is offset by the more extreme thermal reduction in the transition rate out of level 1 , but the measurements presented here suggest that the orbital $T_{1}$ lifetime is only about $20 \mathrm{~ns}$ at $2 \mathrm{~K}$ even for level 1 . Although the spin $T_{1}$ increases with reduced transverse field, these phonon-mediated orbital transitions similarly limit the spin coherence time independent of the field alignment. This picture is consistent with the temperature dependence of the transition linewidth and optical Rabi oscillation decay rate [15].

We have established that the GeV center in diamond combines stable and bright optical transitions with an electronic spin- $\frac{1}{2}$ degree of freedom. Our observation of microwaveoptical double resonance places the $\mathrm{GeV}$ center in a small class of color centers offering microwave control of individual spins $[1,3,27,28]$. It also suggests that a similar microwave access may be possible for the SiV spin system, opening a powerful technique for this family of diamond color centers. Furthermore, the clarity of the Autler-Townes effect highlights the strength and stability of the $\mathrm{GeV}$ optical transitions.

We have shown that, as in the case of the $\mathrm{SiV}, \mathrm{GeV}$ spin coherence is limited by phonon dynamics that depends on temperature. Ongoing efforts to break this barrier for $\mathrm{SiV}$ by microstructures, sub-500 $\mathrm{mK}$ temperature, and strain tuning should also be beneficial for the $\mathrm{GeV}$ electron spin. Combined with efficient optical access to the $\mathrm{GeV}$ electron spin reported here, such developments could enable large-scale quantum networks using GeV centers as quantum memory nodes. The demonstrated optical properties of the $\mathrm{GeV}$ center make this system worth pursuing as a spin-photon interface. 
The authors acknowledge funding from ERC, EU projects (SIQS, DIADEMS, EQUAM), DFG (FOR 1482, FOR 1493, and SFBTR 21), BMBF, USARL/ORISE, DARPA, CUA, ARL, AFOSR, NSF, the Volkswagen Foundation, and Russian
Science Foundation (Grant No. 14-27-00054). We thank Christine Kranz and Gregor Neusser for providing FIB facilities.

P.S. and M.H.M. contributed equally to this work.
[1] A. Gruber, A. Dräbenstedt, C. Tietz, L. Fleury, J. Wrachtrup, and C. von Borczyskowski, Science 276, 2012 (1997).

[2] C. Santori, D. Fattal, J. Vučković, G. S. Solomon, and Y. Yamamoto, Nature (London) 419, 594 (2002).

[3] S.-Y. Lee, M. Widmann, T. Rendler, M. W. Doherty, T. M. Babinec, S. Yang, M. Eyer, P. Siyushev, B. J. M. Hausmann, M. Loncar, Z. Bodrog, A. Gali, N. B. Manson, H. Fedder, and J. Wrachtrup, Nat. Nanotechnol. 8, 487 (2013).

[4] T. Müller, C. Hepp, B. Pingault, E. Neu, S. Gsell, M. Schreck, H. Sternschulte, D. Steinmüller-Nethl, C. Becher, and M. Atatüre, Nat. Commun. 5, 3328 (2014).

[5] F. Jelezko and J. Wrachtrup, J. Phys.: Condens. Matter 16, R1089 (2004).

[6] F. Jelezko and J. Wrachtrup, Phys. Status Solidi A 203, 3207 (2006).

[7] A. Faraon, C. Santori, Z. Huang, V. M. Acosta, and R. G. Beausoleil, Phys. Rev. Lett. 109, 033604 (2012).

[8] K. Nemoto, M. Trupke, S. J. Devitt, A. M. Stephens, B. Scharfenberger, K. Buczak, T. Nöbauer, M. S. Everitt, J. Schmiedmayer, and W. J. Munro, Phys. Rev. X 4, 031022 (2014).

[9] T. Iwasaki, F. Ishibashi, Y. Miyamoto, Y. Doi, S. Kobayashi, T. Miyazaki, K. Tahara, K. D. Jahnke, L. J. Rogers, B. Naydenov, F. Jelezko, S. Yamasaki, S. Nagamachi, T. Inubushi, N. Mizuochi, and M. Hatano, Sci. Rep. 5, 12882 (2015).

[10] Y. N. Palyanov, I. N. Kupriyanov, Y. M. Borzdov, and N. V. Surovtsev, Sci. Rep. 5, 14789 (2015).

[11] E. A. Ekimov, S. G. Lyapin, K. N. Boldyrev, M. V. Kondrin, R. Khmelnitskiy, V. A. Gavva, T. V. Kotereva, and M. N. Popova, JETP Lett. 102, 701 (2016).

[12] L. J. Rogers, K. D. Jahnke, M. H. Metsch, A. Sipahigil, J. M. Binder, T. Teraji, H. Sumiya, J. Isoya, M. D. Lukin, P. Hemmer, and F. Jelezko, Phys. Rev. Lett. 113, 263602 (2014).

[13] B. Pingault, J. N. Becker, C. H. H. Schulte, C. Arend, C. Hepp, T. Godde, A. I. Tartakovskii, M. Markham, C. Becher, and M. Atatüre, Phys. Rev. Lett. 113, 263601 (2014).

[14] K. D. Jahnke, A. Sipahigil, J. M. Binder, M. W. Doherty, M. Metsch, L. J. Rogers, N. B. Manson, M. D. Lukin, and F. Jelezko, New J. Phys. 17, 043011 (2015).

[15] M. K. Bhaskar, D. D. Sukachev, A. Sipahigil, R. E. Evans, M. J. Burek, C. T. Nguyen, L. J. Rogers, P. Siyushev, M. H. Metsch,
H. Park, F. Jelezko, M. Lončar, and M. D. Lukin, Phys. Rev. Lett. 118, 223603 (2017).

[16] Y. N. Palyanov, I. N. Kupriyanov, Y. M. Borzdov, A. F. Khokhryakov, and N. V. Surovtsev, Cryst. Growth Des. 16, 3510 (2016).

[17] J. P. Goss, P. R. Briddon, M. J. Rayson, S. J. Sque, and R. Jones, Phys. Rev. B 72, 035214 (2005).

[18] C. Hepp, T. Müller, V. Waselowski, J. N. Becker, B. Pingault, H. Sternschulte, D. Steinmüller-Nethl, A. Gali, J. R. Maze, M. Atatüre, and C. Becher, Phys. Rev. Lett. 112, 036405 (2014).

[19] L. J. Rogers, K. D. Jahnke, M. W. Doherty, A. Dietrich, L. P. McGuinness, C. Müller, T. Teraji, H. Sumiya, J. Isoya, N. B. Manson, and F. Jelezko, Phys. Rev. B 89, 235101 (2014).

[20] J. P. Hadden, J. P. Harrison, A. C. Stanley-Clarke, L. Marseglia, Y.-L. D. Ho, B. R. Patton, J. L. OBrien, and J. G. Rarity, Appl. Phys. Lett. 97, 241901 (2010).

[21] L. Marseglia, J. P. Hadden, A. C. Stanley-Clarke, J. P. Harrison, B. Patton, Y.-L. D. Ho, B. Naydenov, F. Jelezko, J. Meijer, P. R. Dolan, J. M. Smith, J. G. Rarity, and J. L. O'Brien, Appl. Phys. Lett. 98, 133107 (2011).

[22] S. Castelletto, J. P. Harrison, L. Marseglia, A. C. Stanley-Clarke, B. C. Gibson, B. A. Fairchild, J. P. Hadden, Y.-L. D. Ho, M. P. Hiscocks, K. Ganesan, S. T. Huntington, F. Ladouceur, A. D. Greentree, S. Prawer, J. L. O'Brien, and J. G. Rarity, New J. Phys. 13, 025020 (2011).

[23] E. Neu, M. Agio, and C. Becher, Opt. Express 20, 19956 (2012).

[24] U. Jantzen, A. B. Kurz, D. S. Rudnicki, C. Schäfermeier, K. D. Jahnke, U. L. Andersen, V. A. Davydov, V. N. Agafonov, A. Kubanek, L. J. Rogers, and F. Jelezko, New J. Phys. 18, 073036 (2016).

[25] M. Fleischhauer, A. Imamoglu, and J. P. Marangos, Rev. Mod. Phys. 77, 633 (2005).

[26] J. Kirschner and R. Feder, Phys. Rev. Lett. 42, 1008 (1979).

[27] W. F. Koehl, B. B. Buckley, F. J. Heremans, G. Calusine, and D. D. Awschalom, Nature (London) 479, 84 (2011).

[28] P. Siyushev, K. Xia, R. Reuter, M. Jamali, N. Zhao, N. Yang, C. Duan, N. Kukharchyk, A. D. Wieck, R. Kolesov, and J. Wrachtrup, Nat. Commun. 5, 3895 (2014). 